\title{
Fluorescência da clorofila como uma ferramenta possível para seleção de tolerância à salinidade em girassol ${ }^{1}$
}

\author{
Chlorophyll fluorescence as a possible tool for salt-stress tolerance screening in the \\ sunflower
}

\author{
André Dias de Azevedo Neto ${ }^{2 *}$, Pedro Paulo Amorim Pereira ${ }^{2}$, Danilo Pereira Costa ${ }^{2}$ e Ana Carla Conceição dos \\ Santos $^{2}$
}

\begin{abstract}
Resumo - O efeito do estresse salino de intensidade e duração conhecida nos parâmetros de fluorescência da clorofila em plantas de girassol foi estudado objetivando selecionar genótipos tolerantes e sensíveis à salinidade. O delineamento experimental utilizado foi o inteiramente casualizado em um arranjo fatorial entre 10 genótipos e 2 níveis de salinidade, com quatro repetições. As sementes foram semeadas em areia lavada e, após a germinação, foram transplantadas para vasos contendo solução nutritiva ou solução nutritiva com $100 \mathrm{mM}$ de $\mathrm{NaCl}$, em casa de vegetação. Após 27 dias nessas condições, foram avaliadas a fluorescência basal $\left(\mathrm{F}_{0}\right)$, a eficiência quântica potencial do fotossistema 2 - PS2 $\left(\mathrm{F}_{\mathrm{v}} / \mathrm{F}_{\mathrm{m}}\right)$, a razão $\mathrm{F}_{\mathrm{v}} / \mathrm{F}_{0}$ e a eficiência quântica efetiva do PS2 $(Y)$. Os coeficientes dos "quenching" fotoquímico $\left(\mathrm{q}_{\mathrm{P}}\right)$ e não fotoquímico $\left(\mathrm{q}_{\mathrm{N}}, \mathrm{q}_{\mathrm{CN}}\right.$ e NPQ) também foram calculados. $\mathrm{O}$ estresse salino aumentou $\mathrm{F}_{0}$ e diminuiu $\mathrm{F}_{\mathrm{v}} / \mathrm{F}_{\mathrm{m}}, \mathrm{F}_{\mathrm{v}} / \mathrm{F}_{0}$ e $\mathrm{Y}$, sugerindo injúrias na estrutura dos tilacóides causadas pela salinidade. Esses efeitos foram mais pronunciados no genótipo AG-960 e menos pronunciados no AG-975. O genótipo AG-960 também apresentou os maiores aumentos em $\mathrm{q}_{\mathrm{N}}, \mathrm{q}_{\mathrm{CN}}$ e NPQ, em contraste com o observado para o AG-975. Dessa forma, AG-975 e AG-960 foram caracterizados como tolerante e sensível à salinidade, respectivamente. Os demais genótipos apresentaram tolerância intermediária ao estresse salino. Os resultados indicam que a fluorescência da clorofila pode ser utilizada como uma ferramenta para a seleção de genótipos de girassol tolerantes à salinidade.
\end{abstract}

Palavras-chave - Estresse salino. Helianthus anuus.

\begin{abstract}
The effect of salt stress of known intensity and duration on chlorophyll fluorescence in sunflower plants was studied aiming to identify salt-tolerant and salt-sensitive genotypes. The experimental design was a completely randomized in a factorial arrangement among 10 genotypes and two salt levels, with four replications. Seeds were sown in sand, and after germination were transplanted to plastic pots with nutrient solution or nutrient solution containing $100 \mathrm{mM}$ of $\mathrm{NaCl}$ and placed in a greenhouse. After 27 days, ground fluorescence $\left(\mathrm{F}_{0}\right)$, maximum quantum yield of PS2 $\mathrm{F}_{\mathrm{v}} / \mathrm{F}_{\mathrm{m}}$, the $\mathrm{F}_{\mathrm{v}} / \mathrm{F}_{0}$ ratio and effective quantum yield of photosystem 2 - PS2 (Y) were evaluated. The photochemical (qP) and non-photochemical $\left(\mathrm{q}_{\mathrm{N}}, \mathrm{q}_{\mathrm{CN}}\right.$ e NPQ) coefficients were also calculated. Salt stress increased $F 0$ and reduced $F_{v} / F_{m}, F_{v} / F_{0}$, and $Y$, suggesting salt-induced structural damage in the thylakoid membranes. These effects were more pronounced in genotype AG-960 and lowest in AG-975. The highest increases in qN, qCN and NPQ were also found in genotype AG-960 in contrast with that observed in AG-975. Thus the AG-975 and AG-960 genotypes were characterized as the most salt-tolerant and salt-sensitive, respectively. The other genotypes showed intermediate tolerance to salt stress. Our results suggest that chlorophyll fluorescence could be used as a tool for the screening of sunflower genotypes for salinity tolerance.
\end{abstract}

Key words - Salinity. Helianthus anuus.

\footnotetext{
* Autor para correspondência

${ }^{1}$ Recebido para publicação em 31/08/2010; aprovado em 31/01/2011

Trabalho submetido e selecionado no primeiro Simpósio Brasileiro de Salinidade realizado de 12-15/10/2010 em Fortaleza, Ceará, Brasil; Pesquisa financiada pelo CNPq e realizada no Centro de Ciências Exatas e Tecnológicas (CETEC) da Universidade Federal do Recôncavo da Bahia (UFRB) ${ }^{2}$ Centro de Ciências Exatas e Tecnológicas, Universidade Federal do Recôncavo da Bahia, Campus Universitário, Cruz das Almas-BA, Brasil, 44.380-000, andre@ufrb.edu.br, pedrop87_@hotmail.com,danilocosta_1739@hotmail.com, 1.1.ka@hotmail.com
} 


\section{Introdução}

A salinidade limita a produção agrícola em grandes áreas do mundo. Em áreas salinas ou irrigadas com águas salinas, a maioria das culturas exibe reduções significativas na produção. Visto que a variabilidade genética com respeito à tolerância à salinidade ocorre em girassol (tão bem como em outras espécies), os programas de melhoramento clássico são conduzidos para selecionar genótipos mais tolerantes baseados nas respostas da produção. Contudo, visto que a seleção com base na produção pode ser dispendiosa e demandar um maior espaço de tempo, o uso de parâmetros indiretos baseados nas respostas fisiológicas das plantas à salinidade pode ser mais rápido para a seleção.

Neste cenário, o uso de parâmetros de fluorescência tem sido difundido principalmente no estudo de fotossíntese por ser um método que, além de não destrutivo, permite analisar qualitativa e quantitativamente a absorção e o aproveitamento da energia luminosa através do PS2 e as possíveis relações com a capacidade fotossintética (MOUGET; TREMBLIN, 2002; NETTO et al., 2005).

Mudanças na composição e função do aparato fotossintético das plantas em resposta à salinidade têm sido descritas na literatura recente. Tem sido mostrado que a cinética da fluorescência da clorofila muda em resposta aos estresses abióticos (BAKER, 2008) e tem sido útil na seleção genética de tolerância à salinidade. Dessa forma, esse método pode ser uma excelente ferramenta para a seleção visto que é fácil de ser mensurada, podendo permitir a seleção de um grande número de genótipos em pouco tempo. Nos últimos anos, ela tem sido utilizada com algum sucesso aparente na seleção de algumas culturas para tolerância aos estresses ambientais (BELKHODJA et al., 1994; FLEXAS et al., 2002; RAZAVI et al., 2008). Percival e Fraser (2001) e Glynn et al. (2003) usaram medidas de fluorescência da clorofila como uma ferramenta para diagnosticar a identificação de plantas tolerantes ao sal.

Assim, este trabalho objetivou examinar em detalhes as possíveis mudanças induzidas pelo estresse salino nos parâmetros de fluorescência da clorofila em dez genótipos de girassol, com a finalidade de selecionar genótipos tolerantes e sensíveis à salinidade.

\section{Material e métodos}

Sementes de dez genótipos comerciais de girassol (Helio-358, Albisol-20CL, Albisol-2, NTO-20, AG-960, AG-962, AG-963, AG-967, AG-972 e AG-975) foram semeadas em copos plásticos $(200 \mathrm{~mL})$ contendo areia lavada irrigada diariamente com água destilada, em casa de vegetação. Os valores médios de temperatura, umidade relativa do ar e radiação fotossinteticamente ativa (ao meio dia) foram $27^{\circ} \mathrm{C}, 65 \%$ e $1200 \mu \mathrm{mol} \mathrm{m} \mathrm{s}^{-2}$, respectivamente. Aos oito dias após a emergência, as plântulas foram transferidas para bandejas contendo solução nutritiva de Hoagland e Arnon (1950) diluída 1:2, sob aeração constante, onde permaneceram por mais três dias. Após este período, foram iniciados os tratamentos: controle (cultivo em solução nutritiva) e salino (cultivo em solução nutritiva com $\mathrm{NaCl}$ a $100 \mathrm{mM}$ ). A adição do $\mathrm{NaCl}$ foi realizada gradativamente $(25 \mathrm{mM}$ a cada $24 \mathrm{~h})$, até ser atingida a concentração de $100 \mathrm{mM}$. As plantas permaneceram nestas condições por um período de 27 dias. Diariamente, o nível das soluções foi completado com água destilada e o $\mathrm{pH}$ $(5,5)$ ajustado pela adição de $\mathrm{HCl}$ ou $\mathrm{NaOH}$. A renovação das soluções nutritivas foi realizada semanalmente, até a coleta do material.

A eficiência fotoquímica do PS2 foi mensurada às 5 e $12 \mathrm{~h}$, através de um fluorômetro modulado modelo OSI1FL (ADC Bioscientific Ltd, Hoddesdon, Hertfordshire), no par de folhas mais jovem completamente expandido. Os parâmetros de adaptação ambiental avaliados foram $\mathrm{F}_{0}, \mathrm{~F}_{\mathrm{v}}$ $\mathrm{F}_{\mathrm{m}}, \mathrm{Fv} / \mathrm{F}_{0}$ e Y. Com base no modelo "puddle" de unidade fotossintética, os coeficientes dos "quenching" fotoquímico (qP) e não fotoquímico $\left(\mathrm{q}_{\mathrm{N}}, \mathrm{q}_{\mathrm{CN}}\right.$ e NPQ) também foram calculados (LICHTENTHALER et al., 2005).

O delineamento experimental utilizado foi $\mathrm{o}$ inteiramente casualizado em um arranjo fatorial 10 (genótipos) x 2 (níveis de estresse), com quatro repetições. Os resultados foram submetidos à análise de variância e as médias comparadas pelo teste de Tukey a 5\% de significância.

\section{Resultados e discussão}

As variáveis $\mathrm{F}_{0}$ e $\mathrm{F}_{\mathrm{v}} / \mathrm{F}_{\mathrm{m}}$ relacionadas à fluorescência da clorofila das plantas controle foram semelhantes entre si (TAB. 1). Nas plantas estressadas, $F_{0}$ aumentou significativamente, sendo este efeito mais pronunciado nos genótipos Helio-358, AG-960 e AG-967 (66; 75 e $82 \%$, respectivamente) e menor em AG-975 (32\%). $\mathrm{F}_{0}$ é a fluorescência quando $\mathrm{Q}_{\mathrm{A}}$ (quinona receptora primária de elétrons do PS2) está totalmente oxidada e o centro de reação do PS2 está aberto, situação iminente à ativação das reações fotoquímicas (MOUGET; TREMBLIN, 2002). Dessa forma, $\mathrm{F}_{0}$ é independente dos eventos fotoquímicos e seu aumento pode ser conseqüência de danos no centro de reação do PS2 ou da redução da capacidade de transferência da energia de excitação da antena para o centro de reação (BAKER; ROSENQVST, 2004; BAKER, 2008). Assim, pode ser verificado que, em todos os genótipos, o PS2 foi afetado pela salinidade. 
Tabela 1 - Fluorescência basal $\left(\mathrm{F}_{0}\right)$, eficiência quântica potencial do PS2 $\left(\mathrm{F}_{\mathrm{v}} / \mathrm{F}_{\mathrm{m}}\right)$, razão $\mathrm{F}_{\mathrm{v}} / \mathrm{F}_{0}$, eficiência quântica efetiva do PS2 (Y), coeficiente de dissipação fotoquímica $\left(\mathrm{q}_{\mathrm{p}}\right)$ e coeficientes de dissipação não fotoquímica $\left(\mathrm{q}_{\mathrm{N}}, \mathrm{q}_{\mathrm{CN}}\right.$ e NPQ) de dez genótipos de girassol cultivados em solução nutritiva contendo 0 (controle) ou $100 \mathrm{mM} \mathrm{NaCl}$ (estresse salino)

\begin{tabular}{|c|c|c|c|c|c|c|c|c|}
\hline \multirow{2}{*}{ Genótipos } & \multicolumn{2}{|r|}{$F_{0}$} & \multicolumn{2}{|c|}{$F_{v} / F_{m}$} & \multicolumn{2}{|c|}{$F_{v} / F_{0}$} & \multicolumn{2}{|r|}{$\mathbf{Y}$} \\
\hline & Controle & Estresse & Controle & Estresse & Controle & Estresse & Controle & Estresse \\
\hline Helio-358 & $335 \mathrm{Ba}$ & $556 \mathrm{Aa}(66)$ & $0,803 \mathrm{Aa}$ & $0,595 \mathrm{Bbc}(-26)$ & $4,097 \mathrm{Aa}$ & $1,481 \mathrm{Bbc}(-64)$ & $0,643 \mathrm{Aa}$ & $0,224 \mathrm{Bb}(-65)$ \\
\hline Albisol-20CL & $302 \mathrm{Ba}$ & $431 \mathrm{Ab}(43)$ & 0,790 Aab & $0,641 \mathrm{Ba}(-19)$ & 3,872 Aab & $1,790 \mathrm{Bab}(-54)$ & 0,691 Aa & 0,349 Bab (-49) \\
\hline Albisol-2 & $320 \mathrm{Ba}$ & $442 \mathrm{Ab}(38)$ & $0,726 \mathrm{Ac}$ & $0,561 \mathrm{Bc}(-23)$ & $2,661 \mathrm{Ac}$ & $1,294 \mathrm{Bc}(-51)$ & $0,575 \mathrm{Aa}$ & $0,200 \mathrm{Bb}(-65)$ \\
\hline NTO-20 & $318 \mathrm{Ba}$ & $500 \mathrm{Aab}(57)$ & $0,766 \mathrm{Ab}$ & $0,615 \mathrm{Bb}(-20)$ & $3,291 \mathrm{Ab}$ & $1,607 \mathrm{Bb}(-51)$ & $0,660 \mathrm{Aa}$ & $0,226 \mathrm{Bb}(-66)$ \\
\hline AG-960 & $295 \mathrm{Ba}$ & $518 \mathrm{Aa}(75)$ & 0,775 Aab & $0,586 \mathrm{Bbc}(-24)$ & 3,469 Aab & 1,423 Bbc (-59) & $0,668 \mathrm{Aa}$ & 0,233 Bb (-65) \\
\hline AG-962 & $355 \mathrm{Ba}$ & $521 \mathrm{Aa} \mathrm{(47)}$ & $0,730 \mathrm{Ac}$ & $0,638 \mathrm{Ba}(-13)$ & $2,705 \mathrm{Ac}$ & 1,773 Bab (-34) & 0,579 Aa & 0,301 Bab (-48) \\
\hline AG-963 & $308 \mathrm{Ba}$ & $444 \mathrm{Ab}(44)$ & $0,754 \mathrm{Ab}$ & $0,669 \mathrm{Ba}(-11)$ & $3,066 \mathrm{Abc}$ & $2,071 \mathrm{Ba}(-33)$ & 0,609 Aa & 0,314 Bab (-48) \\
\hline AG-967 & $320 \mathrm{Ba}$ & $581 \mathrm{Aa}(82)$ & $0,758 \mathrm{Ab}$ & $0,628 \mathrm{Bab}(-17)$ & $3,164 \mathrm{Abc}$ & 1,694 Bab (-46) & $0,674 \mathrm{Aa}$ & $0,254 \mathrm{Bb}(-62)$ \\
\hline AG-972 & $319 \mathrm{Ba}$ & $494 \mathrm{Aab}(55)$ & $0,763 \mathrm{Ab}$ & 0,624 Bab (-18) & $3,237 \mathrm{Ab}$ & $1,667 \mathrm{Bb}(-49)$ & $0,625 \mathrm{Aa}$ & $0,243 \mathrm{Bb}(-61)$ \\
\hline AG-975 & $334 \mathrm{Ba}$ & $442 \mathrm{Ab}(32)$ & $0,737 \mathrm{Abc}$ & $0,655 \mathrm{Ba}(-11)$ & $2,851 \mathrm{Abc}$ & $1,914(-33)$ & $0,565 \mathrm{Aa}$ & $0,370 \mathrm{Ba}(-34)$ \\
\hline \multirow[t]{3}{*}{$\mathrm{CV}(\%)$} & \multicolumn{2}{|r|}{8} & \multicolumn{2}{|r|}{5} & \multicolumn{2}{|r|}{10} & \multicolumn{2}{|r|}{14} \\
\hline & \multicolumn{2}{|r|}{$q_{P}$} & \multicolumn{2}{|r|}{$\mathbf{q}_{\mathrm{N}}$} & \multicolumn{2}{|r|}{$\mathbf{q}_{\mathrm{CN}}$} & \multicolumn{2}{|r|}{$\mathrm{N}_{\mathrm{PQ}}$} \\
\hline & Controle & Estresse & Controle & Estresse & Controle & Estresse & Controle & Estresse \\
\hline Helio-358 & $1,073 \mathrm{Aa}$ & $0,862 \mathrm{Aa}(-20)$ & $0,792 \mathrm{Ba}$ & 1,178 Aab (49) & $0,636 \mathrm{Aa}$ & 0,699 Aab (10) & $1,753 \mathrm{Ba}$ & 2,405 Aab (37) \\
\hline Albisol-20CL & $1,070 \mathrm{Aa}$ & $0,907 \mathrm{Aa}(-15)$ & $0,655 \mathrm{Ba}$ & $1,005 \mathrm{Ab}(53)$ & 0,518 Bab & $0,645 \mathrm{ab}(24)$ & $1,088 \mathrm{Bb}$ & 1,586 Abc (46) \\
\hline Albisol-2 & $0,981 \mathrm{Aa}$ & $0,883 \mathrm{Aa}(-10)$ & $0,720 \mathrm{Ba}$ & 1,257 Aab (74) & 0,526 Bab & 0,701 Aab (33) & 1,141 Bab & 2,782 Aab (144) \\
\hline NTO-20 & $1,045 \mathrm{Aa}$ & $0,709 \mathrm{Bb}(-32)$ & 0,617 $\mathrm{Ba}$ & 1,239 Aab (101) & $0,472 \mathrm{Bb}$ & 0,762 Aab (61) & $0,923 \mathrm{Bb}$ & 3,323 Aab (260) \\
\hline AG-960 & $1,047 \mathrm{Aa}$ & 0,985 Аa (-6) & 0,629 Ba & 1,328 $\mathrm{Aa}(111)$ & $0,488 \mathrm{Bb}$ & $0,780 \mathrm{Aa}(60)$ & $0,975 \mathrm{Bb}$ & 3,666 Aa (276) \\
\hline AG-962 & $0,966 \mathrm{Aa}$ & $0,849 \mathrm{Aa}(-12)$ & 0,670 Ba & $1,078 \mathrm{Ab}(61)$ & $0,490 \mathrm{Bb}$ & 0,688 Aab (40) & $1,038 \mathrm{Bb}$ & 2,229 Ab (115) \\
\hline AG-963 & $0,996 \mathrm{Aa}$ & $0,692 \mathrm{Bb}(-30)$ & 0,638 Ba & $0,974 \mathrm{Ab}(53)$ & $0,481 \mathrm{Bb}$ & 0,653 Aab (36) & $0,960 \mathrm{Bb}$ & 2,507 Aab (161) \\
\hline AG-967 & $1,054 \mathrm{Aa}$ & 0,754 Bab (-28) & $0,575 \mathrm{Ba}$ & 1,106 Aab (92) & $0,436 \mathrm{Bb}$ & 0,695 aab (59) & $0,778 \mathrm{Bb}$ & 2,338 aab (200) \\
\hline AG-972 & $1,028 \mathrm{Aa}$ & $0,604 \mathrm{Bb}(-41)$ & 0,674 Ba & 0,937 Abc (39) & 0,514 Aab & $0,586 \mathrm{Abc}(14)$ & $1,065 \mathrm{Ab}$ & 1,246 Ac (17) \\
\hline AG-975 & $1,031 \mathrm{Aa}$ & $0,778 \mathrm{Bab}(-25)$ & $0,769 \mathrm{Aa}$ & 0,799 Ac (4) & $0,568 \mathrm{Aa}$ & $0,523 \mathrm{Ac}(-8)$ & $1,357 \mathrm{Aa}$ & $1,112 \mathrm{Ac}(-18)$ \\
\hline CV (\%) & & 7,5 & & 10 & & 10 & & 23 \\
\hline
\end{tabular}

Médias seguidas de mesmas letras, maiúsculas entre tratamentos salinos dentro de cada genótipo e minúsculas entre genótipos dentro de cada tratamento salino, não diferem entre si pelo teste de Tukey a 5\% de significância. Valores entre parênteses representam os percentuais de aumento (quando positivos) ou de redução (quando negativos) das plantas estressadas em relação às controle

A razão $\mathrm{F}_{\mathrm{v}} / \mathrm{F}_{\mathrm{m}}$ das plantas sob estresse decresceu, em média, $18 \%$ em relação aos controles. Contudo, as maiores reduções foram observadas nos genótipos Helio-358 (26\%) e AG-960 (24\%) e as menores no AG-963 (11\%) e no AG-975 (11\%). A razão $\mathrm{F}_{\mathrm{v}}$ / $\mathrm{F}_{\mathrm{m}}$ é uma estimativa da eficiência quântica máxima da atividade fotoquímica do PS2, quando todos os centros de reação do PS2 estão abertos (BAKER; ROSENQVST, 2004). Essa relação tem sido utilizada para detectar perturbações no sistema fotossintético causada pelo estresse salino, visto que sua diminuição indica um declínio na eficiência fotoquímica do PS2 e um distúrbio ou dano no aparato fotossintético (BELKHODJA et al., 1994; GLYNN et al., 2003; PERCIVAL; FRASER, 2001).
A razão $\mathrm{F}_{\mathrm{v}} / \mathrm{F}_{0}$ também tem sido recomendada para detectar mudanças induzidas pelos estresses (LICHTENTHALER etal., 2005), pois embora contenha a mesma informação básica, amplifica as pequenas variações detectadas pela razão $\mathrm{F}_{\mathrm{v}} / \mathrm{F}_{\mathrm{m}}$, como pode ser observado na comparação entre estas variáveis. Nesse caso, a razão $\mathrm{F}_{\mathrm{v}} / \mathrm{F}_{0}$ nas plantas estressadas decresceu, em média, 47\% em relação aos controles. Entretanto, semelhante ao descrito para $\mathrm{F}_{\mathrm{v}} / \mathrm{F}_{\mathrm{m}}$, as maiores reduções foram observadas em Helio-358 (64\%) e AG-960 (59\%) e as menores em AG-963 (33\%) e AG-975 (33\%).

A eficiência quântica efetiva (Y) indica a fração de energia absorvida pela clorofila associada ao PS2 que foi utilizada em atividade fotoquímica e, como tal, 
informa a quantidade de elétrons transportados, sendo um indicativo da fotossíntese (LICHTENTHALER et al., 2005). O principal fator determinante desta eficiência é a habilidade com que os elétrons são removidos da quinona receptora do PS2, o que está diretamente relacionado com a taxa de consumo de ATP e NADPH, produtos do transporte fotossintético de elétrons (BAKER; ROSENQVST, 2004; NETTO et al., 2005). Nas plantas estressadas, Y diminuiu em média $64 \%$ nos genótipos Helio-358, Albisol-2, NTO-20, AG-960, AG967 e AG-972 e apenas 34\% no AG-975 (TAB. 1).

Quando as plantas são expostas à luz, os centros de reação do PS2 são progressivamente reduzidos, ocorrendo um aumento da fluorescência da clorofila. Em seguida, a fluorescência decai em um fenômeno chamado extinção da fluorescência ("quenching”). Dois parâmetros básicos descrevem a extinção da fluorescência variável da clorofila durante o período de indução da radiação: a extinção fotoquímica e a extinção não fotoquímica da fluorescência variável da clorofila (LICHTENTHALER et al., 2005).

A extinção fotoquímica $\left(\mathrm{q}_{\mathrm{P}}\right)$ é iniciada em função do aumento dos elétrons exportados do PS2 devido à ativação das enzimas envolvidas no metabolismo do carbono e da abertura estomática (BAKER; ROSENQVST, 2004; FLEXAS, 2002). Dessa forma, $q_{p}$ quantifica a capacidade fotoquímica do PS2, e corresponde à fração de centros de reação PS2 abertos. Os dados da Tabela 1 mostram que, sob condições de estresse, a maior redução em $\mathrm{q}_{\mathrm{P}}$ foi observada em AG-972 (41\%), enquanto que em AG$960, \mathrm{q}_{\mathrm{p}}$ não foi afetado pela salinidade. $\mathrm{O}$ aumento dos valores de $q_{p}$, em função da salinidade, em paralelo ao decréscimo da fotossíntese líquida indicam aumento da participação de dreno alternativo de elétrons, como por exemplo a fotorrespiração e o ciclo água-água (HEBER, 2002; SCHREIBER; BILGER, 1987; RIBEIRO et al., 2004). Considerando que o estresse salino reduziu a fotossíntese líquida de todos os genótipos, sendo a maior redução observada no genótipo AG-960 e a menor no AG975 Azevedo Neto et al. (2010), nosso resultado sugere que o genótipo AG-960 não foi capaz de canalizar o fluxo de elétrons para a síntese de NADPH.

A extinção não fotoquímica $\left(\mathrm{q}_{\mathrm{N}}\right)$ reflete a ativação dos processos não fotoquímicos de emissão da fluorescência da clorofila, levando principalmente à dissipação de energia não radiativa, tais como mudanças no gradiente de $\mathrm{pH}$ transtilacoidal, fotoinibição, desconexão dos complexos móveis captadores de luz, formação de zeaxantina, etc. (ROHÁČEK, 2002). Com exceção do AG975 que não foi influenciado pela salinidade, $\mathrm{q}_{\mathrm{N}}$ aumentou em todos os genótipos (TAB. 1), sendo este efeito mais conspícuo em NTO-20 (101\%) e AG-960 (111\%).
Outros processos que levam ao decaimento da fluorescência máxima da clorofila também são freqüentemente utilizados, tais como a extinção não fotoquímica completa da fluorescência da clorofila $\left(\mathrm{q}_{\mathrm{CN}}\right)$ e a extinção não fotoquímica de Stern-Volmer (NPQ). Diferentemente de $\mathrm{q}_{\mathrm{N}}$, tais parâmetros não requerem a determinação da fluorescência mínima sob condições de iluminação (LICHTENTHALER et al., 2005). $\mathrm{q}_{\mathrm{CN}}$ se refere aos processos de dissipação de energia conectados com ambos, extinção não fotoquímica da fluorescência variável $\left(\mathrm{q}_{\mathrm{N}}\right)$ e a mudança relativa de $\mathrm{F}_{0}$. Dessa forma, $\mathrm{q}_{\mathrm{CN}}$ pode ser usado como um indicador da dissipação de energia não radiativa dentro das membranas tilacóides, ou seja, quantifica os processos de dissipação térmica dentro dos complexos PS2. NPQ também quantifica os processos que levam ao decréscimo da fluorescência máxima. Entretanto, NPQ indica a dissipação do excesso de energia radiante na forma de calor nos complexos antena do PS2, ou seja, a fotoproteção induzida pela luz através da dissipação térmica de energia, estando intimamente correlacionado com a formação de zeaxantina (ROHÁČEK; BARTÁK, 1999).

Em nosso trabalho, $\mathrm{q}_{\mathrm{CN}}$ e NPQ (TAB. 1) permaneceram inalterados nos genótipos AG-972 e AG975. Nos demais genótipos, foram observados aumentos significativos dos valores de $\mathrm{q}_{\mathrm{CN}}$ e de NPQ, sendo os mais pronunciados, respectivamente, nos genótipos NTO-20 (61 e 260\%) e AG-960 (60 e 276\%).

\section{Conclusão}

Analisando conjuntamente os dados obtidos podese verificar que os genótipos AG-960 e AG-975 foram os únicos que apresentaram, em todas as variáveis, resultados contrastantes, caracterizando-os como sensível e tolerante ao estresse salino, respectivamente. Dessa forma, nossos resultados indicam que a fluorescência da clorofila pode ser utilizada como uma ferramenta para a seleção de genótipos de girassol tolerantes à salinidade.

\section{Agradecimentos}

Agradecemos ao CNPq pelo apoio financeiro para a realização desta pesquisa.

\section{Referências}

AZEVEDO NETO, A. D. et al. Trocas gasosas em genótipos de girassol sob estresse salino. In: SIMPÓSIO BRASILEIRO DE SALINIDADE, 1., 2010, Fortaleza. Anais... Fortaleza: Wave Media. 1 CD. 
BAKER, N. R. Chlorophyll fluorescence: a probe of photosynthesis in vivo. Annual Review of Plant Biology, v. 59, p. 89-113, 2008.

BAKER, N. R.; ROSENQVST, E. Applications of chlorophyll fluorescence can improve crop production strategies: an examination of future possibilities. Journal of Experimental Botany, Oxford, v. 55, n. 403, p. 1607-1621, 2004.

BELKHODJA, R. et al. Chlorophyll Fluorescence as a Possible Tool for Salinity Tolerance Screening in Barley (Hordeum vulgare L.) Plant Physiology, v. 104, n. 02, p. 667-673, 1994.

FLEXAS, J. et al. Steady-state chlorophyll fluorescence (Fs) measurements as a tool to follow variations of net $\mathrm{CO}_{2}$ assimilation and stomatal conductance during water-stress in $\mathrm{C}_{3}$ plants. Physiologia Plantarum, v. 114, n. 02, p. 231-240, 2002.

GLYNN, P.; FRASER, C.; GILLIAN, A. Foliar salt tolerance of Acer genotypes using chlorophyll fluorescence. Journal of Arboriculture, v. 29, n. 02, p. 61-65, 2003.

HEBER, U. Irrungen, Wirrungen? The Mehler reaction in relation to cyclic electron transport in $\mathrm{C}_{3}$ plants. Photosynthesis Research, v. 73, n. 01-03, p. 223-231, 2002.

HOAGLAND, D. R.; ARNON, D. I. The water-cultured method for growing plants without soil. California: California Agricultural Experiment Station, Circular n ${ }^{\circ} 347$, 1950. 32p.

LICHTENTHALER, H. K. et al. How to correctly determine the different chlorophyll fluorescence parameters and the chlorophyll fluorescence decrease ratio $\mathrm{R}_{\mathrm{Fd}}$ of leaves with the PAM fluorometer. Photosynthetica, v. 43, n. 03, p. 379393, 2005.

MOUGET, J.; TREMBLIN, G. Suitability of the fluorescence monitoring system (FMS, Hansatech) for measurement of photosynthetic characteristics in algae. Aquatic Botany, v.74, p.219-231, 2002.

NETTO, A. T.; CAMPOSTRINI, E.; OLIVEIRA, G. J. et al. Photosynthetic pigments, nitrogen, chlorophyll a fluorescence and SPAD-502 readings in coffee leaves. Scientia Horticulturae, Amsterdan, v. 104, n. 02, p. 199-209, 2005.

PERCIVAL, G. C.; FRASER, G. A. Measurement of the salinity and freezing tolerance of Crataegus genotypes using chlorophyll fluorescence. Journal Arboriculture, v. 27, n. 05, p.233-245, 2001.

RAZAVI, F. et al. Chlorophyll fluorescence as a tool for evaluation of drought stress in strawberry. Photosynthetica, v. 46, n. 04, p. 631-633, 2008.

RIBEIRO, R. V.; MACHADO, E. C.; OLIVEIRA, R. F. Growth and leaf temperature effects on photosynthesis of sweet orange plants infected with Xylella fastidiosa. Plant Pathology, Oxford, v. 53, n. 03, p. 334-340, 2004.

ROHÁČEK, K. Chlorophyll fluorescence parameters: the definitions, photosynthetic meaning, and mutual relationships. Photosynthetica, v. 40, n. 01, p. 13-29, 2002.

ROHÁČEK, K.; BARTÁK, M. Tehcnique of the modulated chlorophyll fluorescence: basic concepts, useful parameters, and some applications. Photosynthetica, v. 37, n. 03, p. 339363, 1999.

SCHREIBER, U.; BILGER, W. 1987. Rapid assessment of stress effects on plant leaves by chlorophyll fluorescence measurements. In: TENHUNEN, J. D.; CATARINO, F. M.; LANGE, O. L.; OECHEL, W. C. Plant response to stress. Berlin: Springer-Verlag, 1987. p.27-53. (NATO ASI Series, Series G). 